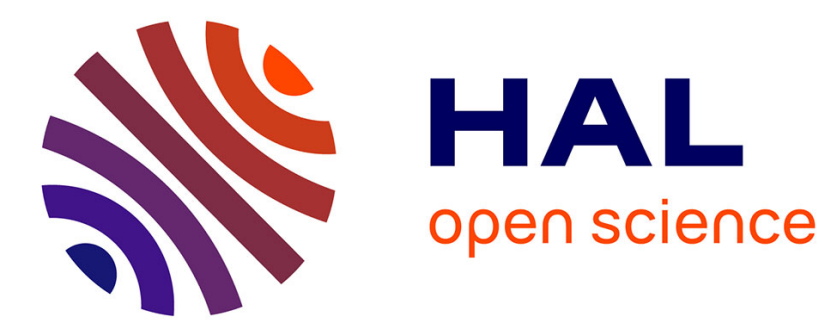

\title{
Dire décalé et sélection de point de vue dans la métalepse
}

Geneviève Salvan

\section{To cite this version:}

Geneviève Salvan. Dire décalé et sélection de point de vue dans la métalepse. Langue française, 2008, Figures et point de vue, 160, pp.70-84. 10.3917/lf.160.0073 . hal-01182187

\section{HAL Id: hal-01182187 \\ https://hal.science/hal-01182187}

Submitted on 6 Aug 2015

HAL is a multi-disciplinary open access archive for the deposit and dissemination of scientific research documents, whether they are published or not. The documents may come from teaching and research institutions in France or abroad, or from public or private research centers.
L'archive ouverte pluridisciplinaire HAL, est destinée au dépôt et à la diffusion de documents scientifiques de niveau recherche, publiés ou non, émanant des établissements d'enseignement et de recherche français ou étrangers, des laboratoires publics ou privés. 


\title{
DIRE DÉCALÉ ET SÉLECTION DE POINT DE VUE DANS LA MÉTALEPSE
}

\author{
Geneviève SALVAN
}

Armand Colin | Langue française

$2008 / 4-n^{\circ} 160$

pages 73 à 87

ISSN 0023-8368

Article disponible en ligne à l'adresse:

http://www.cairn.info/revue-langue-francaise-2008-4-page-73.htm

Pour citer cet article :

SALVAN Geneviève, « Dire décalé et sélection de point de vue dans la métalepse », Langue française, $2008 / 4$ n 160, p. 73-87. DOI : 10.3917/lf.160.0073

Distribution électronique Cairn.info pour Armand Colin.

(C) Armand Colin. Tous droits réservés pour tous pays.

La reproduction ou représentation de cet article, notamment par photocopie, n'est autorisée que dans les limites des conditions générales d'utilisation du site ou, le cas échéant, des conditions générales de la licence souscrite par votre établissement. Toute autre reproduction ou représentation, en tout ou partie, sous quelque forme et de quelque manière que ce soit, est interdite sauf accord préalable et écrit de l'éditeur, en dehors des cas prévus par la législation en vigueur en France. II est précisé que son stockage dans une base de données est également interdit. 


\section{Dire décalé et sélection de point de vue dans la métalepse}

Tantôt classée dans les figures de mots, et plus particulièrement dans les tropes, comme variété de métonymie reposant sur une relation chronologique, tantôt classée dans les figures de pensée comme indirection énonciative, la métalepse est aussi bien convoquée pour rendre compte de l'expression « sentir le sapin » que pour caractériser ces deux vers de Racine, dans lesquels Phèdre avoue à Enone son amour pour Hyppolyte: Quand pourrai-je, au travers d'une noble poussière,/Suivre de l'œil un char fuyant dans la carrière! ${ }^{1}$. Devant cette diversité d'approches et de définitions, il nous paraît nécessaire d'interroger la double tradition qui fonde ces acceptions ${ }^{2}$ (I), avant d'analyser plus en détail la dynamique du raccourci référentiel et énonciatif (II) puis la mise en scène énonciative des points de vue dans la métalepse (III).

\section{LA MÉTALEPSE : VARIANTE DE MÉTONYMIE OU FIGURE AUTONOME?}

La première tradition évoquée, issue de Dumarsais citant Quintilien, la classe comme on l'a dit parmi les tropes comme une variété de métonymie. Elle

1. Fontanier $1997: 128$.

2. En ne présentant que ces deux acceptions, nous en laissons provisoirement une « troisième » de côté, issue de l'acception de Fontanier et qui est à l'origine d'un emploi narratologique chez Genette (2004). Celui-ci définit la métalepse narrative comme transgression des niveaux narratifs «par laquelle le narrateur feint d'entrer (avec ou sans son lecteur) dans l'univers diégétique » (1972: 135, n. 1), et ailleurs, comme «toute intrusion du narrateur ou du narrataire extradiégétique dans l'univers diégétique (ou de personnages diégétiques dans un univers métadiégétique, etc.), ou inversement. » (1992 : 243-246) 
procède par transfert temporel pour exprimer l'antécédent par le conséquent ou le conséquent par l'antécédent :

La métalepse est une sorte de métonymie, par laquelle on explique ce qui suit, pour faire entendre ce qui précède, ou ce qui précède, pour faire entendre ce qui suit : elle ouvre, pour ainsi dire, la porte, dit Quintilien, afin que vous passiez d'une idée à une autre; ex alio in aliud viam proestat, Inst. VIII. 6. C'est l'antécédent pour le conséquent, ou le conséquent pour l'antécédent; et c'est toujours le jeu des idées accessoires dont l'une éveille l'autre. (article "Métalepse » de l'Encyclopédie)

Quintilien la considère même comme l'importation d'un "synonyme impropre ${ }^{3}$ » dans un contexte donné : il justifie ainsi l'inflexion sémantique de entendre pour comprendre, parce que l'audition précède la compréhension et lui est nécessaire. Les deux actions étant liées, la porosité sémantique joue en faveur d'un ajout de sens.

Cette tradition perdure chez Littré : « D'après Dumarsais, figure par laquelle on prend l'antécédent pour le conséquent: il a vécu, pour, il est mort; ou le conséquent pour l'antécédent: nous le pleurons, pour, il est mort». G. Molinié prolonge ces définitions en traitant la métalepse comme une variété de métonymie : c'est selon lui un «type de rapport sémantique qui a reçu une dénomination spécifique dans la tradition rhétorique. Il s'agit ici d'une manipulation sur le jeu avant-après, antécédent-conséquent, préalable-résultat " (Molinié $1986: 212)$.

C'est enfin dans cette tradition que s'inscrit le travail le plus abouti sur la métalepse, celui de M. Bonhomme (1987, $2005: 111$ et 209-210, $2006: 59-60$ et 146-151) qui associe synecdoque et métalepse comme figures péri-métonymiques, définies en termes de " parcours » : la métalepse est « le pendant temporel de la synecdoque dans le cadre métonymique » $(1987: 103)$. Métonymie, synecdoque et métalepse ont un double point commun :

1) Elles produisent, à des fins de refonctionnalisation du discours, des décalages ${ }^{4}$ isotopiques entre des termes associés dans un même univers cognitif.

2) Elles fournissent des arguments empiriques basés sur la structure du réel. (Bonhomme 2005 : 204)

La spécificité de chaque figure vient de «la nature des relations qui [les] fondent : contiguïté pour la métonymie, inclusion (ou contiguiité mérologique) pour la synecdoque, successivité (ou contiguité chronologique) pour la métalepse » (id.). La métalepse assure des transferts temporels soit entre les états successifs d'une même entité dans un univers cognitif, soit entre des entités différentes associées. Trois caractéristiques définissent alors de manière singulière la métalepse :

3. Voir Pier et Schaeffer $2005: 14$.

4. Le terme de décalage signifie ici «transfert » et ne correspond pas à l'emploi que nous en faisons dans le présent article. 
a) elle affecte le devenir d'une même entité de base (du type, Le ver ailé se posait sur les fleurs (Ramuz) : N1 ver "être avant » N1' papillon);

b) elle constitue un cas particulier de métonymie temporelle parce qu'elle concerne des relations temporelles discordantes et non concomitantes (" être avant/après " et non "être en même temps »: pleurer quelqu'un est une métalepse pour il est mort mais pas avoir du nez pour avoir du flair, qui repose sur une simultanéité temporelle et qu'on qualifiera de métonymie, Bonhomme 1987: 89) ;

c) le transfert s'effectue sur le mode du devenir simple (ver pour papillon) ou transformationnel (la sève empoisonnée, pour l'héroïne, cité par Bonhomme $2006: 60)$.

Cette analyse exhaustive et pionnière envisage certes la métalepse dans sa dynamique discursive et son environnement contextuel, mais lui dénie toute autonomie :

Il s'agit d'un trope spécifique fondé sur un trait définitoire constant : le transfert chronologique, ou la réorganisation tropique de l'écoulement du temps. Mais pour être spécifique, la métalepse n'est pas autonome. (Bonhomme 1987 : 87)

La seconde tradition, issue de Fontanier, range la métalepse dans les figures de pensée. Elle n'est «jamais un nom seul mais toujours une proposition » et consiste

à substituer l'expression indirecte à l'expression directe, c'est-à-dire, à faire entendre une chose par une autre, qui la précède, la suit ou l'accompagne, en est un adjoint, une circonstance quelconque, ou enfin $\mathrm{s}^{\prime} \mathrm{y}$ rattache ou s'y rapporte de manière à la rappeler aussitôt à l'esprit. (Fontanier, 1977 : 127-128 ${ }^{5}$ )

La première remarque qui s'impose lorsque l'on confronte les définitions de Dumarsais et de Fontanier, c'est que sous leur différence affichée, elles brillent... par leur ressemblance. Tous deux la considèrent comme un trope, mais le premier la circonscrit au mot, tandis que le second la déconnecte de la métonymie et l'envisage comme une « figure d'expression ».

En effet, la métalepse chez Fontanier est une formulation indirecte qui joue - à la faveur de la polysémie de la particule méta ${ }^{6}$ - soit sur une relation tempo-

5. Chez Fontanier, la métalepse figure dans les tropes « improprement dits », parce qu'elle prend la configuration syntaxique de la proposition et qu'elle exprime une pensée, et non seulement une idée, « avec plus ou moins de déguisement et de détour » (1977 : 109). Son statut figural provient du fait qu'elle est toujours le résultat d'un « choix », d'un « artifice » (id.), et non de la nécessité. Parmi ces tropes "en plusieurs mots », la métalepse est classée aux côtés, entre autres, de l'hyperbole, l'allusion, la litote, dans les «figures d'expression par réflexion », dont les principes sont l'implicite et l'indirection énonciative: «nous la [la pensée] dirons moins que nous ne la ferons concevoir ou deviner, par le rapport des idées énoncées avec celles qui ne le sont pas, et sur lesquelles les premières vont en quelque sorte se réfléchir» (1977: 123).

6. En grec ancien, la préposition meta peut signifier soit «après » (« Meta Khriston prophetes » = «Prophète [venu] après le Christ»), soit «avec » ("O Kyrios meta sou " = «Que le seigneur soit avec toi »), et est de toute manière hautement polysémique. Merci à I. Yocaris pour cet éclairage sémantique. 
relle de succession (une chose est évoquée par une autre chose qui lui est antérieure ou postérieure), soit sur une connexion (un événement principal exprimé par l'une de ses circonstances accessoires) $)^{7}$. C. Fromilhague (1995: 117) indique même que dans la métalepse, c'est tout un contenu propositionnel qui doit souvent être interprété par inférence. À l'appui de cette définition, elle donne le dernier vers bien connu du Dormeur du val : "Tranquille, il a deux trous rouges au côté droit » qui suggère l'état connexe - la mort - et qui ne peut, selon elle, être interprété comme euphémisme, puisqu'il y a " mise en évidence de l'horreur d'une mort violente ${ }^{8} »(i d$.$) . La métalepse manipule la mise en scène du$ référent par focalisation, en même temps que la représentation de la chronologie. C'est ce point de vue qu'adopte enfin N. Laurent (2001: 85) lorsqu'il classe la métalepse dans les figures macrostructurales qui visent la composante référentielle du discours : elle présente une manifestation détournée du réel, en jouant sur un mécanisme d'inférence. Dans cette perspective, la métalepse est finalement rapprochée aussi bien de l'allusion que de l'euphémisme et de la litote, voire de l'allégorie et de l'hypotypose (notamment dans le cas de la métalepse de l'auteur, source de l'extension narratologique chez Genette ${ }^{9}$ ).

$C^{\prime}$ est en tant que figure de mot que la métalepse a été le mieux analysée, lorsqu'elle est clairement adossée à la métonymie : elle tire alors sa spécificité de sa modalité temporelle, par le « délai qu'elle nécessite dans la relation, la coprésence devenant successivité ou consécution» (Bonhomme 1987: 87). Or, manifestement, le processus métaleptique de ré-ordonnancement chronologique - et, plus globalement, associatif - peut s'envisager aussi bien au niveau local du lexème qu'au niveau plus global de l'énoncé et de l'énonciation. En effet, le fait de désigner indirectement, décalage dénominatif qui n'est d'ailleurs pas propre à la métalepse, est lié à une énonciation indirecte qui repose sur l'implicite, puisqu'elle vise à «faire entendre autre chose ».

Cette dimension énonciative et pragmatique permet de saisir la métalepse à différents degrés de production: elle devient nettement macrostructurale lorsque, loin de jouer seulement au niveau local, elle engage la signification

\section{Voir aussi de Boissieu et Garagnon 1997 : 260-261.}

8. On peut se convaincre qu'il ne s'agit pas d'un euphémisme en lisant l'étude qu'en donne ici A. Jaubert: elle rappelle que l'euphémisme est une figure d'atténuation et de convenance et montre qu'il est fondé sur un dialogisme interdiscursif qui en fait une figure de la convention linguistique et sociale. Nulle trace de convention chez Rimbaud...

9. On appelle métalepse de l'auteur la figure par laquelle on attribue au poète le pouvoir d'entrer en personne dans l'univers d'une fiction dont, comme auteur, il est bien la "cause", comme lorsqu'on dit que Virgile "fait mourir Didon » au IV Livre de l'Énéide, en feignant de croire qu'il a lui-même allumé le bûcher de la reine de Carthage. Dans l'article "Métalepse » de l'Encyclopédie, on lit : "On rapporte aussi à cette figure, ces façons de parler de Poëtes, (...), lorsqu'au lieu d'une description, ils nous mettent devant les yeux le fait que la description suppose. (...) Ces façons de parler peuvent aussi être rapportées à l'hypothipose ». Voir également chez Fontanier, la possibilité de rattacher à la métalepse «ce tour non moins hardi que les précédens, par lequel, dans la chaleur de l'enthousiasme ou du sentiment, on abandonne tout à coup le rôle de narrateur pour celui de maître ou d'arbitre souverain, en sorte que, au lieu de raconter simplement une chose qui se fait ou qui est faite, on commande, on ordonne qu'elle se fasse » (1977: 129). 
globale de l'énoncé et les relations énonciatives qui s'y jouent. La configuration syntaxique privilégiée de la figure est certes celle du mot ou du syntagme (1), mais elle peut aussi bien être une proposition, subordonnée (2) ou non dépendante (3) :

(1) Ton cadavre qui boit et qui mange sera

Écrasé, broyé dans la boue. (Hugo, cité dans Bonhomme 2005 : 111)

(2) Gueulard qui ne gueulait plus, le sergent, je le fourrais dans le plâtre. (Michaux, Pléiade, t. 2, $2001: 166)^{10}$

(3) L'Époux d'une jeune beauté

Partait pour l'autre monde. À ses côtés sa femme

Lui criait : Attends-moi, je te suis ; et mon âme,

Aussi bien que la tienne est prête à s'envoler.

Le mari fait seul le voyage. (La Fontaine, Fables, VI, 12, « La jeune veuve »)

L'opération énonciative effectuée dans ces trois exemples varie : désignation en (1), qualification de l'antécédent (ou plus exactement assertion autonome sur celui-ci) en (2), assertion en (3), mais ces configurations répondent toutes trois aux critères de la manipulation chronologique du réel, et de la nécessité d'inférence.

Si l'on abandonne la logique sémantique substitutive pour rendre compte $\mathrm{du}$ fonctionnement de la figure et $\mathrm{qu}^{\prime}$ on adopte une perspective pragmaénonciative, on peut tenter de dégager un noyau commun aux deux perspectives définitionnelles exposées ci-dessus. Celui-ci est à trouver non dans la substitution d'un terme à un autre mais dans le geste énonciatif d'un dire décalé (dire quelque chose pour autre chose ${ }^{11}$ quand il est associé à une formulation indirecte par sélection de point de vue (désormais PDV ${ }^{12}$ ).

Un premier groupe d'exemples issus de Zazie dans le métro ([1959] 1999 : 204 et 233) va nous permettre de préciser notre hypothèse :

(4) (Un dialogue se tient entre Trouscaillon et Fédor Balanovitch, interrompu par ce passage narratif:) Un moustique vola dans la cônerie de la lueur d'un réverbère. Il voulait se réchauffer avant de piquer de nouvelles peaux. Il y réussit. Son corps calciné chut lentement sur l'asphalte jaune.

(5) Jeanne Lalochère la fit monter dans le compartiment.

- Alors tu t'es bien amusée?

- Comme ça.

- T'as vu le métro?

- Non.

- Alors, qu'est-ce que t'as fait?

- J'ai vieilli.

En 4, le lecteur restitue aisément ce qui est tu par le narrateur: la chute du corps calciné du moustique se donne pour l'évocation de sa mort préalable.

10. Je remercie Michèle Monte pour cet exemple.

11. Ce que Fontanier exprime par «faire entendre » et Quintilien par "passer d'une idée à une autre » ou « ouvrir la porte » dans la traduction de Dumarsais.

12. Voir l'introduction d'A. Rabatel pour une définition des PDV. 
L'ellipse narrative ${ }^{13}$ gomme une étape et la fait comprendre, à ce titre elle procède par décalage énonciatif. Mais fait-elle métalepse ? À ce stade de la narration, ce n'est pas sûr. La lecture de la suite du roman nous invite à réinterpréter cette figure. Ainsi, on lit deux pages plus loin :

Trouscaillon se lève à son tour. Il hésite. Les clochards dorment. Le moustique est mort. (1999 : 207)

Trouscaillon, devenu foyer perceptif, rétablit la proposition effacée par le narrateur, en "constatant » un état du monde. L'information redondante pour le lecteur révèle en réalité le changement de focale qui s'est opéré plus haut: d'un PDV surplombant jusqu'à "Il y réussit », le narrateur passe à un PDV réduit, focalisé, sur l'objet. Il se contente d'envisager l'après et fait mine d'ignorer la cause de la chute du moustique. Le décalage énonciatif est associé à la sélection d'un PDV focalisé.

Le jeu des PDV affecte le personnage seul en 5. La mère de Zazie retrouve sa fille après le petit séjour parisien et mouvementé que celle-ci a passé avec son oncle Gabriel. La dernière réplique de Zazie, excipit du roman, «fait entendre » un autre énoncé, que l'on pourrait gloser par «j'ai beaucoup appris sur les choses de la vie, sur les adultes notamment», et l'on perçoit ici nettement le dialogisme de la figure. Deux états successifs de $\mathrm{l}_{2} / \mathrm{e}_{2}{ }^{14}$ sont construits : celui de la petite fille réticente à raconter son séjour parisien, puis celui de la petite fille se racontant dans le même mouvement rétrospectif qu'un narrateur autobiographique. La métalepse manipule les relations temporelles/logiques ${ }^{15}$ certes mais en modifiant bien la nature des PDV.

Notre deuxième partie va maintenant s'attacher à caractériser plus précisément la manipulation que fait subir la métalepse à la composante référentielle et énonciative du discours, et que nous suggérons d'appeler " raccourci » pour désigner à la fois la condensation référentielle et la dynamique énonciative.

\section{LA DYNAMIQUE DU RACCOURCI RÉFÉRENTIEL ET ÉNONCIATIF}

Comment opère l'énonciation métaleptique? La métalepse est appelée transumptio par Quintilien, de transumo «prendre d'un autre » ou « recevoir d'un

13. «(...) non apparition d'une séquence narrative attendue dans un programme parfaitement codé, culturellement déterminé, comme tel épisode traditionnel dans un roman d'aventures, ou récit non fait d'une péripétie pourtant impliquée dans la suite de la narration » (Molinié 1992 : 126). 14. Chez A. Rabatel (voir introduction et 2005 : 126-127), L - le locuteur - est l'instance qui profère un énoncé, dans ses dimensions phonétiques et phatiques ou scripturales, selon un repérage déictique ou selon un repérage indépendant d'ego, hic et nunc, tandis que E désigne l'énonciateur à l'origine du PDV. L1/E1 désigne le syncrétisme du locuteur et de l'énonciateur primaires, $\mathrm{l}_{2} / \mathrm{e}_{2}$ désigne celui du locuteur et de l'énonciateur enchâssés dans l'énoncé du locuteur citant (en situation dialogique).

15. Les deux relations de successivité et de consécution sont d'ailleurs difficiles à dissocier, elles sont souvent en proportion variable, et la relation logique peut l'emporter sur la relation temporelle proprement dite. 
autre ». "L'autre " est donc au cœur de cette figure, ainsi que la notion de passage : ex alio in aliud viam proestat. Fonctionnant sur un principe d'altérité, elle associe deux idées sur la base d'une double manipulation : une des deux idées est effacée au profit de l'autre et le passage entre les deux est assuré par un lien - temporel ou logique - nécessaire certes mais implicite, d'où l'effet de raccourci produit par cette figure.

Le «saut » référentiel peut alors avoir une conséquence sémantique, lorsque les idées sont fortement associées dans l'expérience. La perception du réel et sa mise en mots font alors retour sur la langue pour en modifier la structure sémantique. Ce mécanisme de rétroaction de la parole sur la langue a déjà été souligné par C. Détrie (1998 : 165) à propos des métaphores conventionnelles. Quintilien le pointe en parlant de "synonyme impropre » et Dumarsais le précise lorsqu'il explique ainsi l'évolution sémantique de sors (" sort », «action de tirer au sort » en latin) :

Le partage des biens se faisait souvent, et se fait encore aujourd'hui, en tirant au sort. (...) Le sort précède le partage ; de là vient que sors, en latin, se prend souvent pour le partage même, pour la portion qui est échue en partage ; c'est le nom de l'antécédent qui est donné pour le conséquent. (Ibid.)

La nouvelle dénomination, obtenue par un raccourci qui écrase la successivité des actions au profit du résultat visé (le sort pour le partage), se stabilise en langue, et la métalepse se lexicalise ${ }^{16}$.

Le raccourci manipule la présentation du référent en opérant sur le processus évolutif ou implicatif dans lequel il est engagé. Dans " Martin jura qu'il enterrerait le clerc s'il continuait à les importuner " (Voltaire, Candide in de Boissieu et Garagnon, 1997 : 192), le verbe enterrer désigne par métalepse l'étape subséquente au meurtre. La vision décalée du réel, par décalage temporel ou logique (on retrouve ici la solidarité temporelle et causale, car on ne sort jamais de la formule post hoc propter hoc) est acquise par raccourci. Elle donne à voir un référent à un stade postérieur à celui que le contexte laissait prévoir, et fait l'économie du meurtre, au bénéfice d'ailleurs de la face positive de L1/E1.

Le raccourci dans l'expression métaleptique est obtenu par écrasement de l'épaisseur temporelle propre au processus évolutif d'une notion ou par effacement des frontières entre deux entités. La part du contexte est très importante dans son interprétation, surtout lorsque la métalepse n'opère pas un simple décalage temporel entre les états successifs d'une même entité, mais une manipulation de l'ordre d'apparition de deux notions associées. Dans ce cas, la

16. Au même titre que d'autres figures, la métalepse peut donc jouer un rôle dans l'enrichissement sémantique des unités lexicales et particulièrement dans les phénomènes de polysémie (entendre au sens de comprendre, écouter au sens d'obéir). Ce mécanisme de lexicalisation connaît une version «faible » qu'on pourrait appeler « conventionalisation » : une forme, récurrente dans tel ou tel discours, devient conventionnelle, sans pour autant parvenir au stade de la lexicalisation. Cette conventionalisation peut être idiolectale, ou commune à un groupe de locuteurs. Par exemple, Voltaire semble particulièrement goûter le verbe enterrer pour ses effets métaleptiques. 
métalepse est une lecture possible, mais sans doute pas la seule. Une telle lecture plurielle nous semble possible dans l'exemple suivant :

(...) une sirène à bout portant lui lâcha son barrissement et lui plaqua une seconde entre les épaules un chiffon mouillé, mais c'était une sirène d'usine qui fit seulement couler sur la placette un morne troupeau de Nord Africains. (Julien Gracq, Un Balcon en forêt, 1958 : 11)

Pendant la Seconde Guerre Mondiale, l'aspirant Grange est affecté dans un lieu improbable des Ardennes françaises pour une mission de surveillance. Il arrive à la gare de Moriarmé un soir d'automne, et se laisse surprendre par le cri alarmant d'une sirène. Grange vit alors une expérience foudroyante de peur. Comment interpréter le $\mathrm{SN}$ un chiffon mouillé ?

Interpréter de nombreuses figures revient non seulement à conjecturer les informations référentielles spécifiques qu'elles recèlent, mais surtout à découvrir - au terme de ces conjectures - une référence mieux ajustée aux exigences de chaque contexte. (Bonhomme 2005 : 111)

Manifestement, le SN un chiffon mouillé pose un problème référentiel et son interprétation hésite entre métaphore et métalepse. Dans le premier cas, un chiffon mouillé est rapporté à l'ensemble de l'expression lui plaqua un chiffon mouillé qui devient une métaphore concrète de l'action sudatoire de la peur, ce que suggère notamment la cooccurrence du sujet non animé sirène et des verbes d'action lâcher et plaquer. Dans cette lecture, l'accent est mis sur la figuration du sentiment de peur, bien que saisi à travers son appréhension sensitive. Une autre lecture est possible, engageant plus fortement le potentiel référentiel du SN un chiffon mouillé. Celui-ci désigne un référent tout à fait actuel dans la situation : la chemise du lieutenant est devenue, sous l'effet de la peur engendrée par la sirène, "un chiffon mouillé ». Cette interprétation métaleptique, qui envisage l'état subséquent du référent, s'effectue à la faveur de l'association « chemise »/ "chiffon » déjà stabilisée dans le discours, puisque l'on peut dire d'un vêtement froissé qu'il est «chiffonné ». Le référent reste reconnaissable, mais est appréhendé à un stade postérieur: la logique référentielle est alors moins celle $d^{\prime}$ une vision métamorphique ${ }^{17}$ du réel que celle $d^{\prime}$ une vision décalée. Un changement de point de vue sur l'objet s'est opéré, qui garde une trace de ce parcours, à l'instar de l'anamorphose en peinture. Comme le spectateur en se décalant récupère sur la rétine une autre forme de l'objet, la métalepse en sélectionnant un PDV décalé propose une vision et le parcours qui permet d'y accéder. S'il y a deux lectures possibles non exclusives, c'est sans doute qu'une dimension importante est donnée à la réception de la figure, et que se dessinent les contours d'une lecture plus métaphorique et d'une lecture plus métaleptique. Dans la première interprétation, c'est la peur qui est au centre de la figure, la métaphore en fait une entité animée et concrète (avec une dimension intentionnelle de la peur obtenue par les datifs lui); dans la seconde interprétation, c'est par le biais d'un réel déformé par prospection (chemise « devenue » chiffon mouillé) qu'est appréhendée la sensation de peur. La méta-

17. M. Bonhomme (1987 : 103) rapproche métalepse et métamorphose. 
lepse combine ainsi un raccourci dynamique entre deux choses, ou deux états d'une chose, en n'en exprimant qu'une seule, et une potentialisation référentielle de la forme linguistique : dans l'exemple de Ramuz cité ci-dessus par Bonhomme (Le ver ailé se posait sur les fleurs), le «ver ailé » contient une promesse de "papillon ", le " chiffon mouillé » de Grange contient un souvenir de « chemise». Dans l'exemple ver/papillon, le PDV est évidemment moins perceptible, parce que plus attendu, que dans l'association chemise/chiffon. On perçoit dans cet exemple le rendement référentiel d'une telle figure, et la forte sollicitation du récepteur dans son interprétation.

Si la métalepse renvoie de manière détournée à la réalité visée par le discours (dire quelque chose en visant autre chose qui lui est associé), le détour qu'elle emprunte est de l'ordre de la para-informativité, là où litote et euphémisme visent la sous-informativité et là où l'hyperbole vise la sur-informativité ${ }^{18}$. Parallèlement au "trop » et au "trop peu », place est faite au "à côté », qui subsume le «après » et le «avec ». La métalepse vise à côté pour atteindre sa cible, ce faisant elle établit un lien entre deux signes, dont l'un n'est pas exprimé, mais sollicité par l'apparition de l'autre, dans une vision dynamique.

Un exemple des Fables de La Fontaine, dépassant les limites du syntagme nominal, nous permettra de préciser encore cette notion de raccourci et de l'articuler à la notion de PDV, qui est pour nous le dénominateur commun avec l'emploi narratologique de Genette. L. Spitzer a parfaitement montré comment le fabuliste en ménageant des transitions subtiles réussit à dissimuler la " transition sèche entre la partie didactique et la partie narrative de la fable » (1970 : 170), notamment par l'ellipse narrative et l'allusion. On pourrait ajouter la métalepse, qui joue le même rôle de resserrement temporel entre des actions et entre des états d'objets du discours, envisagés selon différents PDV. En obérant une étape, la métalepse perturbe l'enchaînement des faits au profit d'un seul, qui condense toute la teneur informative. La tendance au resserrement temporel entre les actions trouve alors son point d'orgue dans cet exemple, déjà mentionné :
L'Époux d'une jeune beauté
Partait pour l'autre monde. À ses côtés sa femme
Lui criait : Attends-moi, je te suis ; et mon âme,
Aussi bien que la tienne est prête à s'envoler.

Le mari fait seul le voyage. (La Fontaine, Fables, VI, 12, « La jeune veuve »)

C'est ici tout un contenu antécédent qui est escamoté et interprétable par inférence : le vers souligné présuppose que la jeune femme n'a pas accompagné son époux et le fait d'aller droit au résultat souligne l'enflure en même temps que l'inconséquence du discours direct. Le départ du mari est présenté sous une forme insignifiante. En disant «à côté » ${ }^{19}$, la métalepse préserve pour un temps la neutralité affichée du conteur. Le vers souligné est en effet constatif et exhibe un PDV neutre, momentanément endossé par le locuteur-conteur qui

18. Voir ici même les articles d'A. Jaubert et de B. Verine.

19. A.-M. Paillet-Guth parle de «formulation indirecte» $(2005: 230)$. 
prétend restituer les faits. Dans un second temps néanmoins, cet énoncé constatif « résonne » d'un autre énoncé : il ne peut exister pour lui-même, il n'est qu'un intermédiaire qui "ouvre la porte » (Quintilien) à un autre énoncé, non exprimé, mais restituable : «l'épouse ne part pas ». Cet énoncé négatif, comme tout énoncé négatif, contient des PDV hiérarchiques, dont l'un dément l'autre (pdv 2 = «il est faux de dire que pdv 1 l'épouse part»). Dans « le mari fait seul le voyage », il n'y a qu'un point de vue exprimé, un point de vue simple ${ }^{20}$. Mais ce point de vue simple, autonome, est évidemment l'arbre qui cache la forêt : il dissimule les points de vue hiérarchisés ( $\mathrm{pdv} 2+\mathrm{pdv} 1$ injustifié) qui sont rapportés sans équivoque au locuteur primaire L1/E1, à la faveur de leur convergence avec l'orientation argumentative du début de la fable pris en charge par L1/E1: « La perte d'un époux ne va point sans soupirs./On fait beaucoup de bruit, et puis on se console. [...] On dit qu'on est inconsolable ;/On le dit, mais il n'en est rien » (v. $1-2 ; 12-13)$. La métalepse a sélectionné un PDV décalé, celui de L1/E1 qui ne prendrait en compte que l'étape postérieure (le mari part seul) et ignorerait le point de vue de $1_{2} / e_{2}$ exprimé au DD («je pars avec toi »). L1/ E1 fait comme s'il ne savait pas ce qu'avait dit $\mathrm{l}_{2} / \mathrm{e}_{2}$, en réalité il l'omet, sans le taire complètement. De l'hiatus entre le point de vue exprimé de $1_{2} / \mathrm{e}_{2}$ et le point de vue de L1/E1 naît l'ironie. La métalepse propose un point de vue qui en sous-entend un autre et qui n'acquiert sa pertinence que rapporté à cet autre. L'effacement d'une étape se double de la sélection d'un PDV au détriment d'un autre $^{21}$.

La métalepse réorganise donc le réel par écrasement (chrono) logique, elle court-circuite une étape et combine, à la condensation discursive, la sélection d'un PDV : la figure naît de ce changement de PDV sur l'objet du discours ${ }^{22}$. L'impression de raccourci provient de la variabilité du PDV à partir duquel est construit l'énoncé et on observe deux configurations possibles : 1/un énonciateur épouse momentanément un point de vue et l'abandonne ensuite au profit d'un autre, 2/les points de vue sont rapportés successivement à des énonciateurs distincts. L'exemple (5) illustre la première configuration : les deux points de vue sont rapportés à un énonciateur unique $\left(E 1=\mathrm{e}_{2}\right)$, mais sont attribuables successivement à l'instant $t$, puis à l'instant $t+1$. La seconde configuration, deux points de vue successifs mais hétérogènes sur un même objet du discours, peut être illustrée par l'exemple suivant :

(7) Le noble animal de la race féline attend son adversaire avec courage et dispute chèrement sa vie. Demain quelque chiffonnier achètera une peau électrisable. Que ne fuyait-il donc? (Lautréamont, Chants de Maldoror, cité par Dupriez)

\footnotetext{
20. Un point de vue simple est défini par A. Rabatel comme un point de vue indépendant des autres points de vue. Pour la distinction entre points de vue simple et hiérarchiques, voir Rabatel $2005: 124$.

21. La sélection d'un point de vue est dans les fables "en même temps une prise de position de l'auteur » (Spitzer $1970: 204$ ).

22. Il nous semble que c'est par ce biais qu'on peut unifier le champ de la métalepse, en récupérant la perspective narratologique (voir note 2).
} 
À la désignation chronologiquement décalée du chat par le SN une peau électrisable, s'ajoute, après une confrontation de points de vue (le $\mathrm{pdv} \mathrm{d}^{\prime} \ll$ un rôdeur de barrière » qui aperçoit « un vieux chat musculeux », le pdv du narrateur, voire celui du chat lui-même, responsable de la dénomination «le noble animal de la race féline»), la sélection d'un point de vue décalé : celui de «quelque chiffonnier», volontairement flou, qui ne peut désigner le référent qu'à une étape postérieure à la mort du chat. Ce faisant, la métalepse garde une trace du parcours transformationnel et construit une représentation dynamique du référent.

Le mécanisme d'inférence nécessaire à l'interprétation de la métalepse la rapproche alors du trope implicitatif de C. Kerbrat-Orecchioni ${ }^{23}$. Pour Kerbrat (1986: 116-117), il y a trope implicitatif lorsqu'un contenu non explicité apparaît en contexte comme le véritable contenu à transmettre, le contenu présupposé ou sous-entendu étant alors converti en contenu dénoté. Ce trope constitue selon Kerbrat un moyen d'informer de manière biaisée, sans prendre complètement en charge la responsabilité de l'information présupposée. La métalepse jouant sur les contenus à transmettre relève de ce fonctionnement.

Dans l'exemple de Lautréamont, la mort du chat est présupposée, mais est appréhendée à travers un PDV hétérogène, qui, en l'occurrence ne fait que la constater. Ce PDV constatif sur la réalité, le seul exprimé, est rapporté à un énonciateur qui ne peut prendre en charge l'information présupposée de la mort du chat, laissée volontairement par le narrateur (L1/E1) à la charge du lecteur. La métalepse contribue ainsi aux stratégies de distillation discrétionnaire de l'information par le narrateur.

Dans la métalepse, le dire, en se décalant, est rapporté à un PDV différent. Ce mécanisme nous amène à réévaluer comme métalepse l'exemple suivant, que Bonhomme (2006: 163) donne comme une métonymie: "Jules resta un moment dans la chambre avec Henriette. Puis il descendit, satisfait » (Boudard), précisément parce qu'au PDV omniscient du narrateur succède un PDV focalisé auquel on ne peut rapporter le contenu effacé. Seul le lecteur peut et même doit, par la confrontation des deux phrases, combler le trou dans la narration et opérer le changement de focale. C'est encore ce critère qui permet de saisir la différence avec l'ellipse narrative, figure dans laquelle une étape est gommée sans sélection de PDV (comme dans l'exemple célèbre de l'épilogue de L'Éducation sentimentale ${ }^{24}$ ). La métalepse en effaçant une étape produit quant à elle une déplétion dans la narration : elle lui retranche du volume, elle court-circuite le cours des choses par bifurcation brusque. Le raccourci obtenu est alors aussi une ligne de fuite, puisque le point de vue est souvent aussitôt abandonné.

La métalepse sélectionne un point de vue aux dépens d'un autre sur un objet de discours, et nécessite une inférence pour parvenir à une interprétation

23. C. Kerbrat-Orecchioni revisite la théorie de tropes dans son ouvrage sur l'implicite (1986). Elle étend la définition de la rhétorique classique en élargissant les tropes aux figures de pensée.

24. Classiques Garnier, $1984: 425$. 
figurale de l'énoncé : c'est la mise en scène des points de vue dans la métalepse et son rendement pragmatique que nous abordons maintenant.

\section{LA MISE EN SCÈNE ÉNONCIATIVE DES POINTS DE VUE DANS LA MÉTALEPSE}

L'énonciateur métaleptique amène le destinataire à inférer quelque chose à partir d'autre chose : "si je dis X, c'est pour faire entendre Y qui lui est lié par une relation temporelle/logique nécessaire ». La désignation par raccourci se double d'un point de vue décalé, parfois rétrospectif, le plus souvent anticipant, comme dans l'exemple suivant :

(8) Le lait tombe; adieu veau, vache, cochon, couvée ;

La dame de ces biens, quittant d'un œil marri

Sa fortune ainsi répandue,

Va s'excuser à son mari

En grand danger d'être battue.

(La Fontaine, Fables, VII, 9, « La Laitière et le pot au lait », v. 23-27)

Pour désigner par «fortune répandue» ce qui est référentiellement observable, à savoir le lait perdu, l'énonciateur doit se placer dans un 'à côté' référentiel: pas un 'après' chronologique mais un 'après' rapporté au fantasme de la laitière. La métalepse ajoute au raccourci référentiel lait répandu / fortune perdue ${ }^{25}$ la sélection du point de vue fantasmatique de la laitière : "sa fortune ", qui est d'ailleurs ainsi nommée au moment où elle en a le moins le potentiel - c'est l'ironie du sort - désigne «le lait qu'elle voyait déjà comme devant lui rapporter une fortune ». Le référent est visé depuis le foyer perceptif du personnage et l'on retrouve les deux caractéristiques de la métalepse, condensation référentielle et sélection d'un point de vue décalé. La métalepse souligne alors dans la fable le pathétique de la situation en condensant le processus d'un « devenir contrarié ».

Le locuteur-conteur avait introduit en amont le point de vue du personnage, par les vers suivants: "Notre laitière ainsi troussée/Comptait déjà dans sa pensée/Tout le prix de son lait, en employait l'argent,/Achetait cent d'œufs, faisait triple couvée ;/La chose allait à bien par son soin diligent. » (v. 7-11) Le passage qui suit ces vers est formellement un discours direct («disait-elle») mais correspond plutôt en termes de contenu à des pensées rapportées, les châteaux en Espagne de la fermière ${ }^{26}$ :

Il m'est, disait-elle, facile,

D'élever des poulets autour de ma maison :

25. À la métalepse s'ajoute l'hypallage simple qui redistribue syntaxiquement le qualificatif « répandue » et condense les points de vue sur l'objet (voir sur ce sujet Gaudin-Bordes \& Salvan 2008 a).

26. On sait qu'à l'époque classique, les pensées rapportées sont actualisées comme du discours rapporté (avec des incises du type « disait-elle à soi-même » ou « disais-je en moi-même »), voir Jaubert 2005. 
Le Renard sera bien habile,

S'il ne m'en laisse assez pour avoir un cochon.

Le porc à s'engraisser coûtera peu de son ;

Il était quand je l'eus de grosseur raisonnable. (v. 11-17)

Les désignations successives actualisent les différents points de vue : celui du locuteur avec le SN neutre «son lait » (v. 9), puis celui présomptueux de la laitière, dont le décalage temporel - prospectif et rétrospectif fantasmatiques est présent dans le texte (v. 16-17). L'apparition de formes verbales au passé dans le DD manifeste en outre un double décentrage temporel : la temporalité du personnage $l_{2} / e_{2}$ dont le fabuliste rapporte les propos, puis à l'intérieur des propos rapportés, le passé fictif de l'acquisition du porc ${ }^{27}$. La métalepse sert alors, comme très souvent chez La Fontaine, à exprimer «l'ironie du sort », par le biais de la déceptivité.

La métalepse apporte parfois sa contribution, avec d'autres figures, à des stratégies discursives plus globales de stigmatisation d'espoir déçu et d'attente trompée. Soit cette fable, qui joue de manière récurrente sur les désignations référentielles, et où la métalepse côtoie la périphrase et la paradiastole ${ }^{28}$ :

(9) Le long d'un clair ruisseau buvait une Colombe,

Quand sur l'eau se penchant une Fourmi y tombe ;

Et dans cet Océan l'on eût vu la Fourmi

S'efforcer, mais en vain, de regagner la rive.

La Colombe aussitôt usa de charité :

Un brin d'herbe dans l'eau par elle étant jeté,

Ce fut un promontoire où la Fourmi arrive.

Elle se sauve ; et là-dessus

Passe un certain Croquant qui marchait les pieds nus.

Ce Croquant par hasard avait une arbalète.

Dès qu'il voit l'Oiseau de Vénus,

Il le croit en son pot, et déjà lui fait fête.

Tandis qu'à le tuer mon Villageois s'apprête,

La Fourmi le pique au talon.

Le Vilain retourne la tête.

La Colombe l'entend, part, et tire de long.

Le soupé du croquant avec elle s'envole :

Point de Pigeon pour une obole.

(La Fontaine, Fables, II, 12, « La Colombe et la fourmi », je souligne)

Il y a dans cette fable au moins quatre points de vue auxquels rapporter les différentes désignations : un PDV «neutre » (celui du conteur, responsable de la dénomination descriptive "eau», et du SN "mon villageois»), un PDV minorant sur le monde (celui de la colombe, à qui rapporter les syntagmes « clair ruisseau » et «brin d'herbe »), un PDV majorant sur le monde (celui de

27. Qu'on pourrait interpréter comme les propos que la laitière s'imagine tenir et qu'elle rapporte sans marquage dans son propre discours (du DD doublement enchâssé).

28. Sur cette figure de «nomination antagoniste », voir Gaudin-Bordes \& Salvan 2008 b. 
la fourmi responsable de " océan » et de " promontoire $^{29}$ »), un PDV de convoitise (celui du croquant). Le fabuliste estompe momentanément l'ancrage référentiel en usant de la périphrase "Oiseau de vénus " ${ }^{30}$, et l'ironie point de l'hiatus entre le point de vue de L1/E1 et celui du croquant. Ce détour dénominatif lance la vision quasi hallucinatoire du croquant qui est filée dans la suite. La métalepse porte sur le syntagme "le soupé du croquant », qui re-désigne la colombe dans la perspective transformationnelle que lui assigne le croquant (« il le croit en son pot»). Mais ce qui est original ici, c'est que des points de vue hétérogènes sur le même objet sont construits par deux expressions référentielles coprésentes dans le discours : d'une part «le soupé du croquant» qui manifeste un point de vue modal décalé et d'autre part, le pronom «elle » désignant sans détour le référent immédiatement appréhendable dans la réalité (« la colombe )). La dissimilation référentielle du même objet, soutenue par le choc des genres des deux expressions grammaticales, entre ce que l'oiseau aurait pu devenir et ce qu'il est, souligne ironiquement la frustration du paysan. La métalepse est alors exemplifiée sur le fil du discours : la vision déformante, la sélection d'un PDV décalé («le soupé du croquant») sont tenus avec le PDV origine (« elle»). La fable déplie donc le jeu de construction des figures d'énonciateurs mises en scène par la métalepse et leur interaction dans la construction référentielle de l'objet de discours.

L'interaction des PDV dans la métalepse en fait une figure privilégiée de la narration, mais en disant partiellement et partialement, elle relève $d^{\prime}$ 'une vision analytique de la réalité et peut être mise à profit également dans le cadre d'une interaction verbale, comme le dialogue théâtral. Les stratégies discursives s'appuient alors nettement sur ses capacités présuppositionnelles, révélant la partialité affichée de certains personnages. Ainsi, dans cet échange entre Arlequin et Silvia, Marivaux tire parti de la figure en s'appuyant sur le raccourci qu'elle suppose :

\section{ARLEQUIN}

Un Domestique là-bas m'a dit d'entrer ici, et qu'on allait avertir mon beaupère qui était avec ma femme.

SILVIA

Vous voulez dire Monsieur Orgon et sa fille, sans doute, Monsieur? ARLEQUIN

Eh oui, mon beau-père et ma femme, autant vaut ; je viens pour épouser, et ils m'attendent pour être mariés, cela est convenu, il ne manque plus que la cérémonie, qui est une bagatelle. (Marivaux, Le jeu de l'amour et du hasard, I, 7)

29. Promontoire étant en langue classique un synonyme de cap, ce n'est pas par sa dénotation qu'il est ici intéressant, mais par sa connotation. Le dictionnaire de l'Académie remarque que le terme promontoire est "daté » : " ce mot n'a guère d'usage qu'en parlant de la Géographie ancienne : dans la Géographie moderne, on dit Cap». Promontoire connote donc l'antiquité et ajoute au signifié «qui s'avance » celui de "élevé ».

30. Selon Bonhomme 2005 : 112, la périphrase, comme d'ailleurs la litote, se caractérise par un masquage référentiel. 
Derrière l'humour d'une vision anticipante, Arlequin tente en réalité par ce raccourci de transformer ses désirs en réalité. La métalepse est ici le support de la disjonction entre le point de vue de Silvia et celui d'Arlequin.

L'approche pragma-énonciative à laquelle nous conviait Alain Rabatel dans le présent dossier a permis de pointer la spécificité de la métalepse et son autonomie dans le champ figural. La visée référentielle condensée et décalée, associée à l'interaction particulière des points de vue dans la figure, met à jour la construction des figures d'énonciateurs dans le discours et la dynamique propre à leur construction. Le rendement pragmatique de la figure est multiple : elle tire profit de la possible éphémère des points de vue pour ébaucher des lignes de fuite narratives, elle met en scène leur partialité pour les démasquer et les dénoncer, ou encore elle pointe leur disjonction et fait naître l'ironie.

\section{Références bibliographiques}

BoIsSieu DE J.-L., GARAGnON A.-M. (1997), Commentaires stylistiques, Paris, SEDES.

BONHOMME M. (1987), «Un trope temporel méconnu: la métalepse », Le Français moderne n 55, p. 84- 104.

- (2005), Pragmatique des figures du discours, Paris, Honoré Champion.

- (2006), Le discours métonymique, Paris, Honoré Champion.

DÉTRIE C. (1998), «Comme dit l'autre... L'autre, le corps et le réel dans le processus métaphorique »,

L'autre en discours, Montpellier, Presses de l'Université Paul Valéry, p. I65-I87.

DUMARSAIS (1786), Article « Métalepse » de l'Encyclopédie.

DUPRIEZ (1980), Gradus. Les procédés littéraires, Paris, I0/I8.

FONTANIER P. (1977), Les figures du discours, Paris, Flammarion, collection « Champs ».

Fromilhague C. (1995), Les figures de style, Paris, Nathan Université, collection « I 28 ».

GAUDIN-BORDES L., SALVAN G. (2008 a), «Le sens en marche: le cas de l'hypallage », L'Information grammaticale $n^{\circ} 116$, p. 15-19.

- (2008 b), « La paradiastole : un mot pour un autre », Actes du colloque La reformulation, Rennes, 19.

20 mai 2006, à paraître.

GenetTE G. (1972), Figures III, Paris, Seuil, collection « Tel ».

- (1992), Nouveau discours du récit, Paris, Seuil.

- (2004), Métalepse. De la figure à la fiction, Paris, Seuil.

GRACQ J. (1958), Un balcon en forêt, Paris, Corti.

JAUBERT A. (2005), «Le monologue intérieur. Pragmatique de l'infra-dire et dérèglementation

syntaxique », La langue littéraire et le changement linguistique, F. Berlan (éd.), Paris, PUPS, p. 419-429.

KeRBRAT-ORECCHIONI C. (1986), L'implicite, Paris, A. Colin.

LAURENT N. (200 I), Initiation à la stylistique, Paris, Hachette Supérieur, collection « Ancrages 》.

MOLINIÉ G. (1986), Éléments de stylistique française, Paris, PUF.

- (1992), Dictionnaire de rhétorique, Paris, Le Livre de Poche.

Paillet-Guth A.-M. (2005), « Aspects de l'ironie dans les Fables de La Fontaine », La langue, le style, le sens, Études offertes à Anne-Marie Garagnon, Paris, Éditions L'improviste, p. 229-237.

PeRELMAN C., OlBRECHTS-TYTECA (1958), Traité de l'argumentation, Paris, PUF.

PIER J., SCHAEFFER J.-M. (éds.) (2005), Métalepses : entorses au pacte de la représentation, Paris, Éditions de l'EHESS.

QUENEAU R. (1999), Zazie dans le métro, Paris, Gallimard, collection « Folio junior ».

RABATEL A. (2005), « La part de l'énonciateur dans la construction interactionnelle des points de vue »,

Marges linguistiques, $n^{\circ}$ 9, Saint-Chamas, M. LM.S. éditeur, p. I I5-136.

SPITZER L. (1970), Études de style, Paris, Gallimard. 